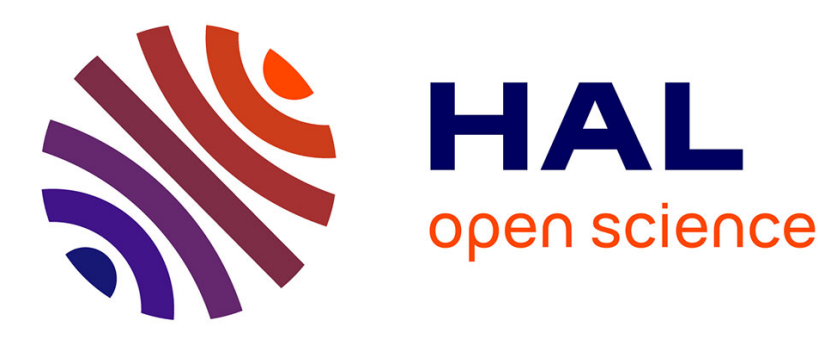

\title{
Wetting and orientation of catalytic Janus colloids at the surface of water
}

Xiaolu Wang, Martin In, Christophe Blanc, Paolo Malgaretti, Maurizio Nobili, Antonio Stocco

\section{- To cite this version:}

Xiaolu Wang, Martin In, Christophe Blanc, Paolo Malgaretti, Maurizio Nobili, et al.. Wetting and orientation of catalytic Janus colloids at the surface of water. Faraday Discussions, 2016, 191, pp.305324. 10.1039/c6fd00025h . hal-01397538

\section{HAL Id: hal-01397538 \\ https://hal.science/hal-01397538}

Submitted on 11 Mar 2019

HAL is a multi-disciplinary open access archive for the deposit and dissemination of scientific research documents, whether they are published or not. The documents may come from teaching and research institutions in France or abroad, or from public or private research centers.
L'archive ouverte pluridisciplinaire HAL, est destinée au dépôt et à la diffusion de documents scientifiques de niveau recherche, publiés ou non, émanant des établissements d'enseignement et de recherche français ou étrangers, des laboratoires publics ou privés. 


\section{Wetting and orientation of catalytic Janus colloids at the surface of water}

Xiaolu Wang, ${ }^{1}$ Martin In, ${ }^{1}$ Christophe Blanc, ${ }^{1}$ Paolo Malgaretti, ${ }^{2}$ Maurizio Nobili ${ }^{1}$ and Antonio Stocco*, ${ }^{1}$

${ }^{1}$ Laboratoire Charles Coulomb (L2C), UMR 5221 CNRS-Univ. Montpellier , Montpellier F-34095, France.

${ }^{2}$ Max Planck Institüt für Intelligente Systeme, Heisenbergstrasse 3, D-70569 Stuttgart, Germany and IV Institüt für Theoretische Physik, Universität Stuttgart, Pfaffenwaldrign 57, D-70569 Stuttgart, Germany.

*E-mail: Antonio.Stocco@umontpellier.fr 


\section{ABSTRACT}

Janus colloidal particles show remarkable properties in terms of surface activity, self-assembly and wetting. Moreover they can perform autonomous motion if they can chemically react with the liquid in which they are immersed.

In order to understand the self-propelled motion of catalytic Janus colloids at the air-water interface, wetting and the orientation of the catalytic surface are important properties to be investigated. Wetting plays a central role in active motion since it determines the contact between fuel and catalytic surface as well as the efficiency of transduction of chemical reaction into motion. Active motion is not expected to occur either when the catalytic face is completely out of the aqueous phase or when the Janus boundaries are parallel to the interfacial plane. The design of a Janus colloid possessing two hydrophilic faces is required to allow the catalytic face to react with the fuel (e.g. $\mathrm{H}_{2} \mathrm{O}_{2}$ for Platinum) in water and to permit some rotational freedom of the Janus colloid in order to generate propulsion parallel to the interfacial plane.

Here, we discuss some theoretical aspects that should be accounted when studying Janus colloids at the surface of water. The free energy of ideal Janus colloidal particles at the interface is modeled as a function of the immersion depth and the particle orientation. Analytical expressions of the energy profiles are established. Energetic aspects are then discussed in relation to the particle ability to rotate at the interface. By introducing contact angle hysteresis we describe how the effects of contact line pinning modifies the scenario described in the ideal case. Experimental observations of the contact angle hysteresis of Janus colloids at the interface reveal the effect of pinning; and orientations of silica particles half covered with a platinum layer at the interface do not comply with the ideal scenarios. Experimental observations suggest that Janus colloids at the fluid interface behave as kinetically driven system, where the contact line motion over defects decorating the Janus faces rules the orientation and rotational diffusion of the particle.

\section{INTRODUCTION}

In ancient Roman religion, the god depicted with two faces called Janus is the god of the beginnings (i.e. the first month of the year January is name after Janus), journeys and transitions: future and past, war and peace; and being the god of the doorway and gate can look both inside and outside. A Janus colloidal particle as Janus can also undergo (interfacial) transitions and be both inside and outside a fluid, when attached to the fluid interface.

In the recent literature, several investigations reported Janus colloidal particles of different size, shape and functionalities. ${ }^{1,2,3}$ Both faces of a Janus particle can be now designed and fabricated with a multiplicity of chemical and physical functions. New self-assemblies, interactions and wetting properties have been realized..$^{2,3,4,5,6}$ If one of the faces is hydrophilic and the other is hydrophobic, Janus particles can combine the properties of surface activity and self-assembly of molecular surfactants with the wetting properties of solids. Hence the behavior of Janus particles at the fluid interface reflects those features, which have an impact in the field of thin films, foams and emulsions. $^{7,8,9}$

If one of the faces of the Janus particle is chemically reactive and the other is inert, the particle can perform autonomous motion in presence of a reactant fuel. ${ }^{10,11}$ Self-generated phoretic fields are responsible for the particle self-propulsion. For instance, the platinum face of catalytic Janus colloids 
can transform dissolved hydrogen peroxide into water and oxygen. Hence, a concentration gradient of reactants and products between the reactive face and inert one will be established and a self-propulsion will be generated. ${ }^{12}$ Janus colloids become active particles able to transform chemical energy into motion, mimicking the behavior of biological microswimmers. This enhanced particle motion has been exploited to perform tasks such as transporting cargo and purifying water by adsorbing or reducing contaminants. ${ }^{13,14}$

Recently Wang et al. ${ }^{15}$, on the experimental side, and Malgaretti et al. ${ }^{16}$, on the theoretical side, have investigated the motion of catalytic Janus colloids at the air-water interface in order to elucidate the role of partial wetting confinement in the active motion realization. Janus colloids were trapped at the interface and both the persistence length and velocity of the self-propelled motion within the interfacial plane were enhanced with respect to the bulk. ${ }^{15}$ The realization of active motion at the interface is governed by wetting and by the orientation of the Janus colloid. The catalytic face reacts with hydrogen peroxide only if it is wetted by the aqueous solution. Directional motion parallel to the interface would also be strongly hindered if the catalytic face is oriented parallel to the interface.

For active colloidal systems, the persistence of the directional motion is ruled by the Brownian rotational diffusion which acts towards randomizing the motion directions. ${ }^{12,17}$ For particles attached onto a fluid interface, rotational diffusion coefficients vary significantly with respect to the bulk rotational diffusion coefficient $\left(D_{r, B}=k T /\left(8 \pi \eta R^{3}\right)\right.$, where $k T$ is the thermal agitation energy, $\eta$ is the dynamic viscosity of the liquid and $R$ is the radius of the particle). ${ }^{18}$ The enhanced persistence of the motion of Janus colloids at the air-water interface was explained in terms of a slowing down of rotational motion at the air-water interface due to partial wetting. ${ }^{15}$

In this article, we focus our attention on wetting and orientation of Janus colloids at the air-water interface in order to elucidate on the active motion recently observed. ${ }^{15}$ Theoretical and experimental results presented here are obtained for catalytic Janus particles but in the absence of fuel. These findings shed light on the fundamental aspects which need to be clarified in order to understand the behavior of Janus particles both in passive and active conditions. Therefore our results will serve as a starting point for more detailed numerical as well as experimental studies aiming at understanding how the activity of the particle breaks the equilibrium picture as well as how the details of fluid rearrangement close to particle surface ${ }^{19}$ can modulate the overall transport properties of the particle.

The structure of the text is the following. In the next section, we discuss some theoretical aspects that should be accounted when studying Janus colloids trapped at the fluid interface. Analytical expression of the free energy profile as a function of the orientation of the Janus colloid is reported; and the role of hydrodynamic rotational friction and surface defects is discussed. Experimental methods and results obtained for spherical silica colloids half covered by platinum are described and discussed in section 3 and 4. Section 5 is dedicated to the significance of our investigations for self-propelled Janus colloids trapped at the surface of water.

\section{THEORETICAL ASPECTS}

In this section we describe the free energy of an isolated Janus colloid as a function of the immersion depth and the orientation at the air-water interface accounting only for the interfacial energy terms. Interfacial frictions of Janus colloids at the interface are also introduced together with the role of surface defects. Some criteria for the design of Janus particles will be also discussed. 


\subsection{FREE ENERGY OF A JANUS COLLOID AS A FUNCTION OF THE IMMERSION DEPTH}

Here we consider a spherical particle of radius $R$ possessing two hemispherical faces of equal area. The particle surface is smooth and homogeneous in each hemispherical face. Within these assumptions we neglect for the moment the effect of pining of the contact line. Moreover, we do not discuss the effect of line tension, since it does not affect significantly the energy of Janus colloids in the micrometer scale $\gamma R^{2} \gg k_{L} R$ (where $\gamma=10^{-1}-10^{-2} \mathrm{~N} / \mathrm{m}$ is a typical interfacial tension), for accepted values of the line tension $k_{L}=10^{-9}-10^{-12} \mathrm{~N}^{20-22}$ Line tension effect starts becoming significant for nanoparticles of few tens of nanometers or lower. Using similar scaling arguments, gravity can be neglected here.

The description of the free energy of such Janus particle at the interface is different from the one of a bare colloid, because the two faces of the Janus particle, in general, possess different surface energies. Free energy of a Janus particle will therefore depend not only on the immersion depth but also on the orientation of the two faces and the area ratio of the two faces. ${ }^{5}$

We begin our analysis considering the case of Janus colloid with two distinct faces of equal area $\left(2 \pi R^{2}\right)$ at the air-water (A-W) interface under the assumption of a flat fluid interface and with the boundary of two faces parallel to the interfacial plane. Both faces are denoted as the $P$ and $S$ face respectively, see Fig. 1. Such conditions, that has been firstly considered by Ondarçuhu et al., ${ }^{5}$ allows us to both introduce the basic concepts and to use them as a benchmark to compare with the regimes where the constraint on the orientation of the Janus particle is relaxed. Following Ondarcuhu et al., 5 we encode in the particle free energy $E(\alpha)$ all the contribution to the free energy of the system that depend on particle properties and/or particle immersion depth $z$. By introducing the contact angle $\alpha=\arccos (-z / R)$ (see Fig. 1) the particle energy reads:

If $\alpha<90^{\circ}$,

$$
E(\alpha)=2 \pi R^{2}\left[\gamma_{P-W}+\gamma_{S-W}(\cos \alpha)+\gamma_{S-A}(1-\cos \alpha)-\frac{1}{2} \gamma_{A-W} \sin ^{2} \alpha\right]
$$

If $\alpha>90^{\circ}$,

$$
E(\alpha)=2 \pi R^{2}\left[\gamma_{S-A}+\gamma_{P-A}(-\cos \alpha)+\gamma_{P-W}(1+\cos \alpha)-\frac{1}{2} \gamma_{\mathrm{A}-\mathrm{W}} \sin ^{2} \alpha\right]
$$

A

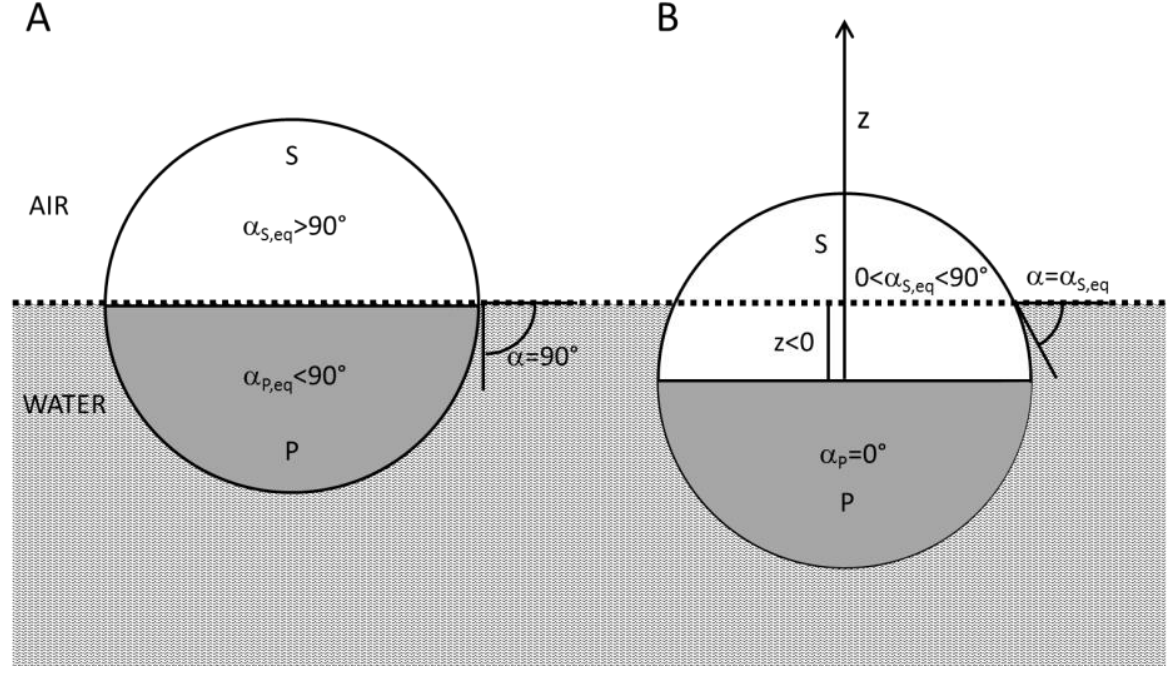

Fig. 1 Sketch of an amphiphilic Janus colloid (A) and a double hydrophilic Janus colloid (i.e. possessing two hydrophilic faces) (B) at the air-water interface. 
where $\gamma_{S-A}$ is the $S$ face-air interfacial tension, $\gamma_{S-W}$ is the $S$ face-water interfacial tension, $\gamma_{P-A}$ is the $P$ face-air interfacial tension, $\gamma_{P-W}$ is the $P$ face-water interfacial tension, and $\gamma_{A-W}$ is the air-water interfacial tension : $\cos \alpha_{S, e q}=\left(\gamma_{S-A}-\gamma_{S-W}\right) / \gamma_{A-W}$ and $\cos \alpha_{P, e q}=\left(\gamma_{P-A}-\gamma_{P-W}\right) / \gamma_{A-W}$. In Fig. 2 we plot some profiles calculated for typical values of the interfacial tensions.

Configurations of single Janus particles at a fluid interface have been discussed by Casagrande et al. ${ }^{6}$. When the two faces possess very distinct contact angles: the hydrophilic face $(P) \alpha_{P, e q}<90^{\circ}$ and the hydrophobic one $(S) \alpha_{S, e q}>90^{\circ}$, the equilibrium contact is $90^{\circ}$, see Figure 1 (A) and Figure 2 (A). In this configuration, the hydrophobic face is immersed in the hydrophobic medium and the hydrophilic face is immersed in the hydrophilic medium. The immersion depth is just determined by minimization of the area of unfavorable contact and none of the faces respects its equilibrium contact angle: the contact line wets the Janus boundary with the unique geometry of a quadruple line, where four different media meet. Both faces pull the Janus particle towards their side and the free energy profile shows a singularity at the minimum as shown in Fig. 2.

When both the $P$ and $S$ faces prefer to be wetted by water, $\alpha_{P, e q}<90^{\circ}$ and $\alpha_{S, e q}<90^{\circ}$, the Janus colloid can be defined as double hydrophilic and the configuration of the Janus will be similar to the one of a bare colloid, see Figure 1 (B). However, also in this case the free energy profile shows a discontinuity in the first derivative when the fluid interface crosses the Janus boundary. The energy profile of a double hydrophilic Janus colloid possessing $\alpha_{S, e q}=21^{\circ}, \alpha_{P, e q}=0^{\circ}$ is also shown in Fig. 2(A). It is worth noting that the adsorption energy for a double hydrophilic particle $E(\alpha=0)-E\left(\alpha_{\mathrm{eq}}\right)=0.3 \times 10^{6} \mathrm{kT}$ is much lower than the amphiphilic case showing a value of about $10^{8} \mathrm{kT}$.

The double hydrophilic system is representative of platinum-silica colloids able to perform autonomous motion when immersed in a hydrogen peroxide solution. ${ }^{15,23}$ Considering the latter system and plugging literature values of the interfacial tensions (silica-air $\gamma_{S-A}=58.6 \mathrm{mN} / \mathrm{m}$, silica-water $\gamma_{S-W}=$ $25 \mathrm{mN} / \mathrm{m} ;{ }^{24}$ platinum-air $\gamma_{P-A}=153 \mathrm{mN} / \mathrm{m}$ and platinum-water $\gamma_{P-W}=110 \mathrm{mN} / \mathrm{m}^{25}$ ) we calculate the free energy profiles in the two possible configurations when the Janus boundaries are parallel to the interface. The first one is the configuration describing the platinum face $(P)$ oriented towards the water and the second one is the one with the platinum face oriented towards the air. The two energy profiles were calculated using Eqn. 1 and 2 are shown in Fig. 2(B). 

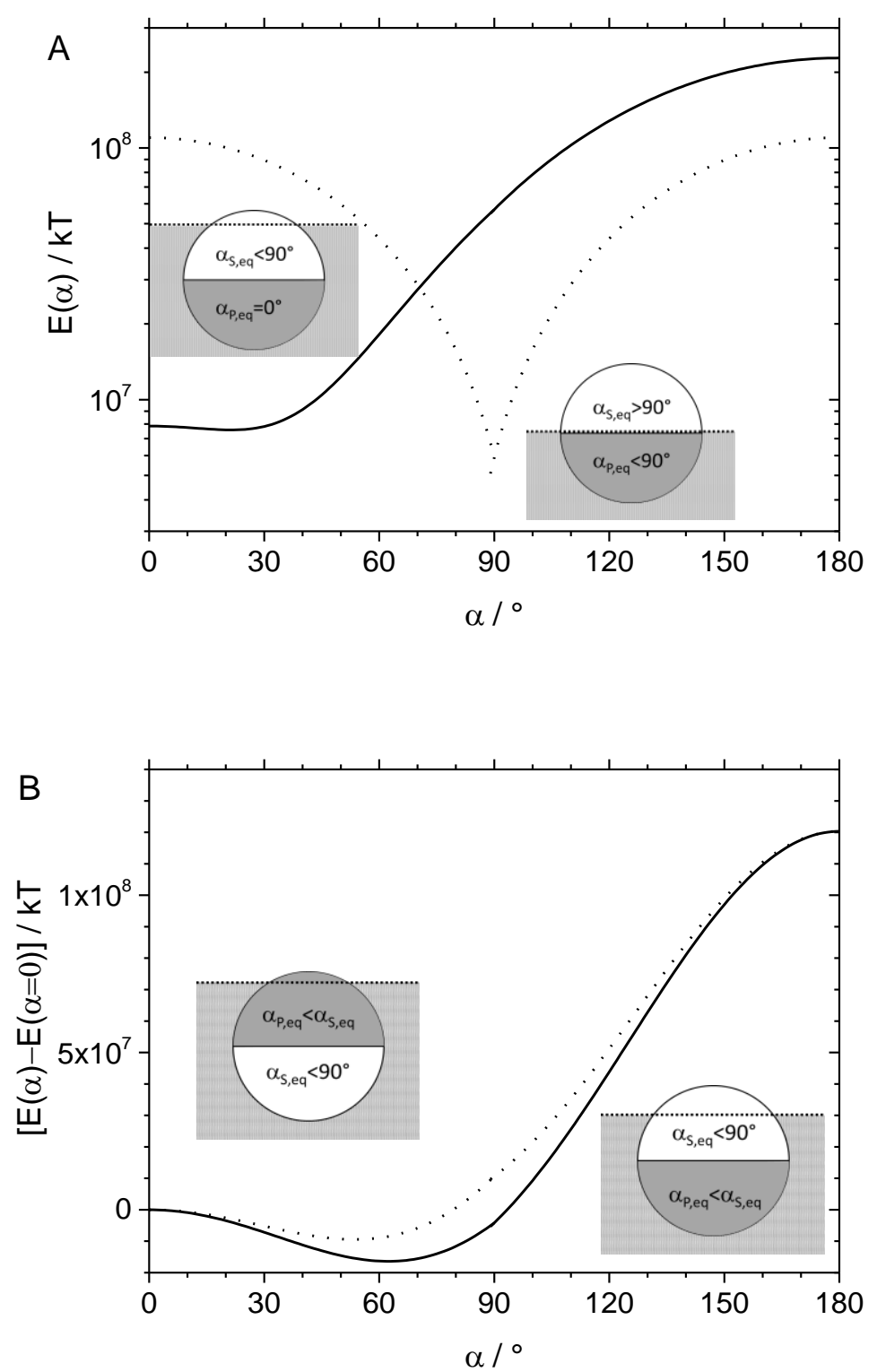

Fig. 2 (A) Energy as a function of the contact angle $\alpha$ for an amphiphilic (dotted line) and a double hydrophilic Janus colloid (solid line). Here the energy profiles were calculated using Eqn.1 and 2: $R=1 \mu \mathrm{m}, \gamma_{A-W}=72.5$ $\mathrm{mN} / \mathrm{m}$, (1) solid curve: $\alpha_{S, e q}=21^{\circ}, \alpha_{P, e q}=0^{\circ} \quad\left(\gamma_{S-A}=\gamma_{A-W}=72.5 \mathrm{mN} / \mathrm{m}, \gamma_{S-W}=5 \mathrm{mN} / \mathrm{m}, \gamma_{P-A}=\gamma_{A-W}\right.$, $\left.\gamma_{P-W}=0\right)$; (2) dashed curve: $\alpha_{S, e q}=114^{\circ}, \alpha_{P, e q}=66^{\circ}\left(\gamma_{S-A}=\gamma_{P-W}=20 \mathrm{mN} / \mathrm{m}\right.$ and $\gamma_{P-A}=\gamma_{S-W}=50$ $\mathrm{mN} / \mathrm{m})$.

(B) Energy profile of a platinum-silica Janus colloid oriented with the platinum face towards air (dotted line) and with the silica face towards air (solid line), $\mathrm{E}(\alpha=0)=2.12 \times 10^{8} \mathrm{kT}$.

In this example, given that the contact angle of platinum is smaller than the one of silica, ${ }^{24,26,25}$ the energy profile with the platinum oriented towards water leads to a smaller free energy as compared to the case with the opposite orientation. The minimum of energy corresponds to the contact angle of Janus colloid which is just the silica equilibrium contact angle $\alpha_{S, e q}=61^{\circ}$, and platinum fully immersed in water. 
Note that if the Janus colloid is unable to change its orientation, stable contact angles may be found whenever equilibrium on the triple line will be satisfied. This occurs not only at $\alpha=61^{\circ}$ on the silica face but also at $\alpha_{P, e q}=52^{\circ}$ on the platinum face.

Having assumed that the Janus boundary remains parallel to the interface, we did not consider yet any change of orientation of the faces of the Janus particle. The calculations presented just above are actually valid for any orientation that prevents the Janus boundary from intersecting the interface.

\subsection{FREE ENERGY OF A JANUS COLLOID AS A FUNCTION OF THE ORIENTATION}

Now we start to investigate the change of the free energy as a function of the orientation of a Janus colloid when the Janus boundary is not parallel to the interface. Here we consider the case of a double hydrophilic colloid and assume that the equilibrium contact angle $\alpha_{e q}$ is the same along the three phase contact line and that the two Janus faces possess the same area $\left(2 \pi R^{2}\right)$. The orientation is defined by the angle $\beta$ between the interface normal (i.e. $z$-axis) and the Janus boundary normal $\hat{n}$, Fig. 3 .

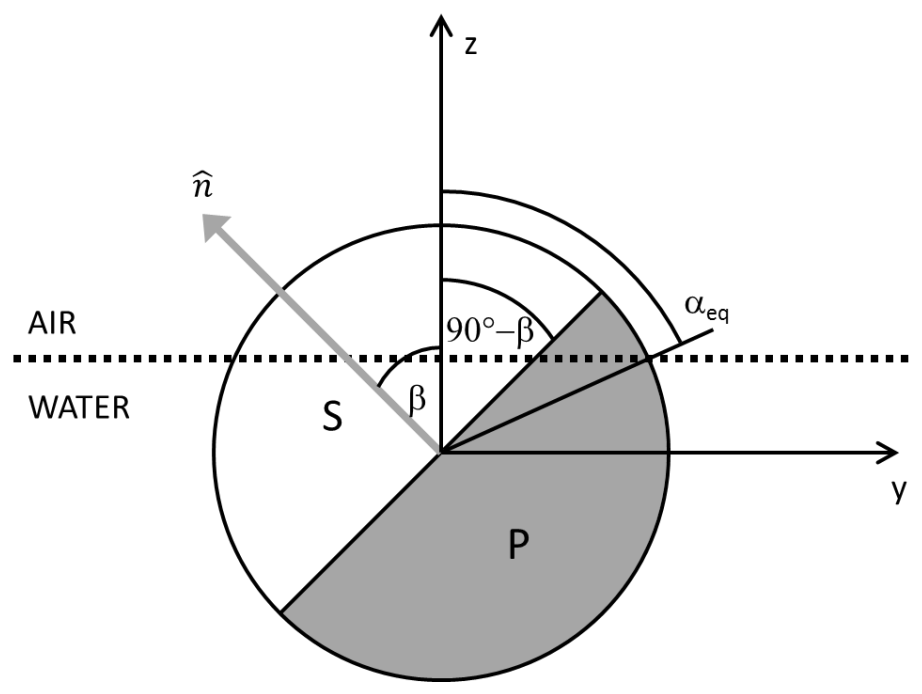

Fig. 3 Schematic representation of Janus colloid at air-water interface with the Janus boundary normal formed an angle $\beta$ with $z$-axis.

Even though the free energy of spheroidal particles has been calculated analytically, ${ }^{27,28}$ so far the free energy landscape as a function of the orientation of Janus particles has been calculated only numerically or using computer simulations. ${ }^{29-31}$ Here, we present an analytical expression of the free energy assuming a flat interface at a given equilibrium contact angle $\alpha=\alpha_{e q}$. In this view our model can be considered the extension of the works of Ondarcuhu et al. to the case in which the Janus equator is not parallel to the interface.

In the range $0<90^{\circ}-\beta<\alpha_{e q}$, the free energy $E(\beta)=E_{T}-\gamma_{A-W} A_{I}$ can be written as:

$$
E(\beta)=\gamma_{S-A} A_{S-A}+\gamma_{P-A} A_{P-A}+\gamma_{S-W} A_{S-W}+\gamma_{P-W} A_{P-W}-\pi R^{2} \gamma_{A-W} \sin ^{2} \alpha_{e q}
$$

Where $A_{i}$ are the area of $S$ or $P$ face immersed either in air or water. The conservation of total area of the particle and conservation of the area of each Janus face yield : 


$$
\begin{aligned}
& A_{S-A}+A_{P-A}=2 \pi R^{2}\left(1-\cos \alpha_{e q}\right) \\
& A_{S-W}+A_{P-W}=2 \pi R^{2}\left(1+\cos \alpha_{e q}\right) \\
& A_{S-A}+A_{S-W}=A_{P-A}+A_{P-W}=2 \pi R^{2}
\end{aligned}
$$

A fourth relation is obtained by expressing for instance $A_{P-A}$ in spherical coordinates $(r, \theta, \varphi)$ :

$$
\begin{gathered}
A_{P-A}=R^{2} \int_{\theta=\frac{\pi}{2}-\beta}^{\alpha_{e q}} \int_{\varphi=\arcsin (1 / \tan \beta \tan \theta)}^{\pi-\arcsin (1 / \tan \beta \tan \theta)} \sin \theta \cdot d \theta d \varphi \\
A_{P-A}=\pi R^{2}\left(1-\cos \alpha_{e q}\right)+2 R^{2}\left[\cos \alpha_{e q} \arcsin \left(\operatorname{cotan} \beta \cdot \operatorname{cotan} \alpha_{e q}\right)-\arctan \frac{\cos \beta}{\sqrt{\sin ^{2} \beta-\cos ^{2} \alpha_{e q}}}\right]
\end{gathered}
$$

Fig. 4 shows two profiles calculated for two different immersion depth corresponding to the equilibrium contact angle of silica and platinum $\alpha_{e q}=61^{\circ}$ and $52^{\circ}$ (Fig. 2). Three different regimes are distinguished depending on the $\beta$ dependence of $E$. The first regime (I) starts at $90^{\circ}-\beta=\alpha_{e q}$, when all $\mathrm{P}$ face is wetted by water. This regime describes a minimum energy plateau and it covers the range $\alpha_{e q}<90^{\circ}-\beta<180^{\circ}-\alpha_{e q}$. It means that the orientation angles corresponding to the energy minimum covers a range of $180^{\circ}-2 \alpha_{e q}$, i.e. the smaller $\alpha_{e q}$ the larger the range! The following regime (II) $180^{\circ}-\alpha_{e q}<90^{\circ}-\beta<180^{\circ}+\alpha_{e q}$ covers a range of $2 \alpha_{e q}$. Both $P$ and $S$ faces are partially wetted both. The last regime (III) corresponds to the range of maximum energy with the $S$ face completely wetted by water and the $P$ face towards the air.

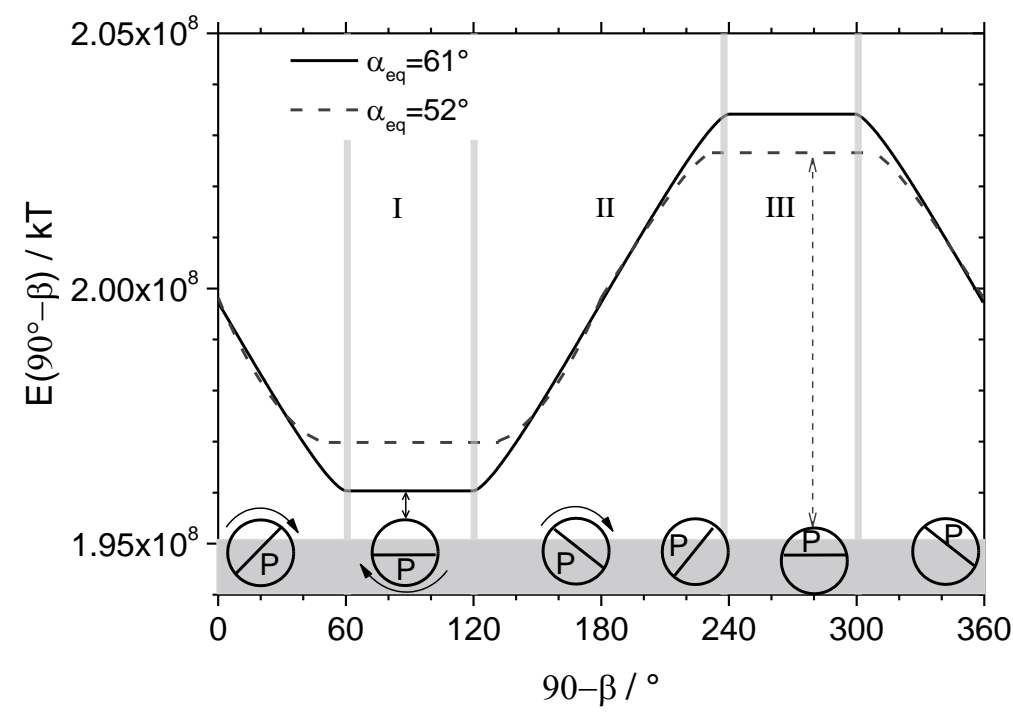

Fig. 4 Two profiles calculated for two different vertical positions corresponding to the equilibrium contact angle of silica $\left(\alpha_{e q}=61^{\circ}\right.$, solid line) and platinum $\left(\alpha_{e q}=52^{\circ}\right.$, dashed line). The scheme at the bottom shows the Janus colloid rotation at air-water interface. I, II, III correspond to the three regimes described in the text. 
As sketched in Fig. 4, isolated Janus colloids may rotate only in the range of angle $\alpha_{e q}<90^{\circ}-\beta<180^{\circ}-\alpha_{e q}$ where the free energy is at the minimum. A further rotation would correspond to an energy cost of about a million of $k T$ for typical values of the interfacial tensions. ${ }^{24,25}$ It is also worth pointing out that upon a rotation, the Janus colloid may change its equilibrium contact angle $\alpha_{e q}$ to minimize its energy. Hence, a Janus colloid oriented with the $P$ face towards water, which starts rotating with an equilibrium contact angle as the one of the $S$ face (see the solid up-down arrow, Fig. 4) may switch to the equilibrium contact angle of the $P$ face (see the dashed up down arrow, Fig. 4) when all the $S$ face is wetted by water.

Note that in all these calculations we assume that the air-water interface keeps flat. With this assumption the Janus colloid will be subjected to a torque in the regime II since the actual contact angle on one of the faces is not the equilibrium contact angle. If the contact line lies on both silica and platinum surfaces, a complete analysis would show a dipolar distortion of the water surface to satisfy the two different contact angles. ${ }^{29-31}$ This would yield a restoring torque for colloids with non-pinned lines. Deformation of the fluid interfaces due to non-equilibrium orientations have been investigated by computer simulations in the minimum surface energy requirement. ${ }^{29}$ In the micrometer scale, accounting for the fluid deformation, one calculates slightly lower energy than assuming a flat interface. Hence, our model provides the free energy only when the energy associated to the deformation of the interface remains small when compared to the total energy.

\subsection{ROLE OF SURFACE DEFECTS}

When accounting for roughness and inhomogeneity of the surface, the ideal scenario described above may change significantly. Here we assume that the fluid interface remains macroscopically flat but the three phase contact line can be deformed due to nanometric defects. Weak or strong pinning of the contact line on a single defect may occur depending on the amplitude of the defect force $F_{D}=$ $F_{l}\left(\mathrm{y}_{\mathrm{m}}-\mathrm{y}_{\mathrm{d}}\right)$ with respect to the restoring force $F_{R}=\mathrm{k}\left(\mathrm{y}_{\mathrm{m}}-\mathrm{y}_{\mathrm{L}}\right)$ of the liquid tail $\left(F_{l}\right.$ is the defect force function, $\mathrm{y}_{\mathrm{m}}$ is the actual position of the line, $\mathrm{y}_{\mathrm{d}}$ is the defect position, $k$ is the spring constant of the restoring force and $y_{L}$ is the position of the contact line in absence of the defect). ${ }^{32,33,34}$

For weak defects, $F_{l} / k$ is small and no hysteresis of the contact angle is expected. Nonetheless the contact line may show some displacement, whose mean squared amplitude is $\left\langle\Delta y^{2}>\sim R \xi\right.$, where $\xi$ is the correlation length of the defect and $R$ is the radius of the particle. ${ }^{32}$ If $\xi=1 \mathrm{~nm}$ and $R=1 \mu \mathrm{m}$, the amplitude of the contact line displacement is about $30 \mathrm{~nm}$. Note that the latter value is much greater than the amplitude of thermal capillary waves ${ }^{35}(\approx 0.2 \mathrm{~nm})$ or the characteristic displacement in the Molecular-kinetic theory of partial wetting $(0.1-1 \mathrm{~nm}){ }^{36}$

For strong defects, $F_{l} / k$ is large and contact angle hysteresis occurs. The contact line is pinned on the defects and only if enough energy is injected into the system, a line displacement will occur towards a new position for which $F_{D}=F_{R}$. The limiting values of stable macroscopic contact angles are the advancing $\alpha_{A}$ and receding $\alpha_{R}$ contact angles. For a spherical colloid, $\alpha_{A}$ is the angle reached after a displacement of the contact line resulting in an increase of the colloid wetted area; whereas $\alpha_{R}$ is the angle reached after a displacement of the contact line resulting in a decrease of the wetted area of the colloid. A useful expression of the contact angle hysteresis for isolated and non-interacting defects reported for structured hydrophobic surfaces made of micropillars is: ${ }^{37}$

$\Delta \cos \alpha=\cos \alpha_{R}-\cos \alpha_{A} \approx \frac{1}{4} a \phi \ln \left(\frac{\pi}{\phi}\right)$ 
where $a$ is a dimensionless geometrical parameter and $\phi$ is the density of the micropillars or defects. Equation 9 may be also interpreted in terms of the influence of surface roughness defects in the contact angle hysteresis.

Thus, for non-interacting defects one expects an increase of the contact angle hysteresis if the density of defects increases.

In the following we discuss how the free energy profile $E(\alpha)$ would be affected by surface defects. We consider only the case of Janus colloid faces decorated by topographical defects, i.e. roughness. The only consequence of a defect is to increase the surface area. The simplest case are cylindrical defects of height $h_{d}$ and radius $R_{d}$. Those defects decorate each face of the Janus colloid with a defect surface fraction $\phi_{d}=\mathrm{n}_{\mathrm{d}} \pi R_{d}^{2} /\left(2 \pi \mathrm{R}^{2}\right)$, where $\mathrm{n}_{\mathrm{d}}$ is the number of defects. The modified energy profile becomes:

If $\alpha<90^{\circ}$,

$$
E(\alpha)=2 \pi R^{2}\left[\left(1+2 \phi_{d, P} \frac{h_{d, P}}{R_{d, P}}\right) \gamma_{P-W}+\left(1+2 \phi_{d, S} \frac{h_{d, S}}{R_{d, S}}\right) \gamma_{S-W}(\cos \alpha)+\left(1+2 \phi_{d, S} \frac{h_{d, S}}{R_{d, S}}\right) \gamma_{S-A}(1-\cos \alpha)-\frac{1}{2} \gamma_{A-W} \sin ^{2} \alpha\right]
$$

If $\alpha>90^{\circ}$,

$$
E(\alpha)=2 \pi R^{2}\left[\left(1+2 \phi_{d, S} \frac{h_{d, S}}{R_{d, S}}\right) \gamma_{S-A}+\left(1+2 \phi_{d, P} \frac{h_{d, P}}{R_{d, P}}\right) \gamma_{P-A}(-\cos \alpha)+\left(1+2 \phi_{d, P} \frac{h_{d, P}}{R_{d, P}}\right) \gamma_{P-W}(1+\cos \alpha)-\frac{1}{2} \gamma_{\mathrm{A}-\mathrm{W}} \sin ^{2} \alpha\right]
$$

The effect of increasing defect density is shown in Fig. 5, where we consider a scenario accounting for defects only in the $S$ face and $h_{d} / R_{d}=1$. As expected, the presence of defects increases the energy but more importantly it deeply modifies its $\alpha$ dependence. As the specific area of the colloid increases the extra energy cost due to colloid-water contact get closer and closer to the energy gain from the reduction of air-water contact and this progressively shift the $E(\alpha)$ minimum and make it more shallow. One may compare the angles of the energy minima calculated in eq. 10 and shown in Fig. 5 with of the Wenzel equilibrium contact angle $\alpha_{W}$ for rough surfaces characterized by a ratio $r$ of the actual area and the geometric area. ${ }^{38} \alpha_{W}=\operatorname{acos}\left(\frac{\gamma_{S-A}-\gamma_{S-\mathrm{W}}}{\gamma_{A-W}} r\right)=\operatorname{acos}\left[\frac{\gamma_{S-A}-\gamma_{S-W}}{\gamma_{A-W}}\left(1+2 \phi_{d, s} \frac{h_{d, s}}{R_{d, S}}\right)\right]$ agrees well with the values calculated in eq. 10: $\alpha_{W}=61^{\circ}$ for $\phi_{d, S}=0, \alpha_{W}=54^{\circ}$ for $\phi_{d, S}=0.1, \alpha_{W}=47^{\circ}$ for $\phi_{d, S}=0.2$ and $\alpha_{W}=29^{\circ}$ for $\phi_{d, S}=0.4$, see Fig. 5 .

However it is important to recall that the main consequence of the presence of strong defects is still the pinning of the contact line. Hence, it may be very slow for the Janus colloid to find the equilibrium position. In the next section kinetic aspects related to the interfacial friction of the Janus colloid rotational motion are discussed. 


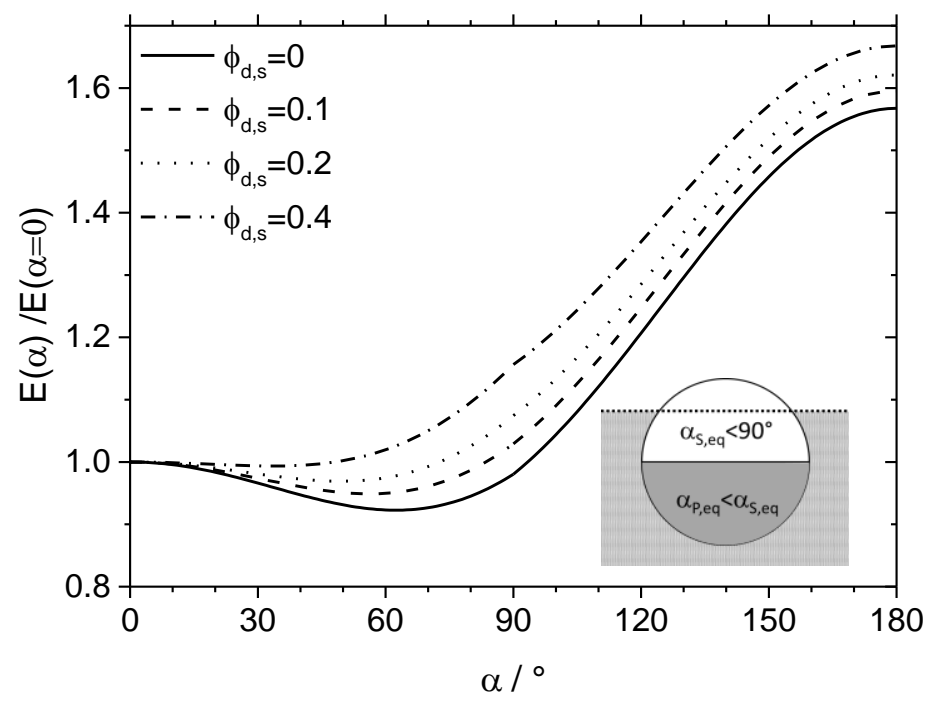

Figure 5 Free energy profile accounting for topographical cylindrical defects of given area fraction $\phi_{d, S}$ and $\phi_{d, P}=$ $0, h_{d}=R_{d}$. Solid line in the figure corresponds to the solid line in Fig. 2 (B).

\subsection{INTERFACIAL ROTATIONAL FRICTIONS}

The rotational friction (about an axis parallel to the interface) of a colloid trapped at the surface of water is related to the hydrodynamic dissipation described in partial wetting when dealing with the motion of a liquid drop approaching its equilibrium contact angle. ${ }^{39}$ In both cases, the dissipation is determined by the relative motion of the liquid and the solid at the contact line. A no-slip condition would lead to an infinite dissipation. ${ }^{39}$ Hence a colloidal particle does not rotate at all in no-slip conditions! However as in partial wetting dynamics, a slip length or a molecular cut-off can be introduced to account for large but finite dissipation.

Recent numerical studies have characterized the diffusion of homogneous ${ }^{40}$ and Janus ${ }^{19}$ nanoparticles adsorbed at fluid-fluid interface. On the other hand, very few theoretical predictions on the rotational motion even of bare spherical particles partially immersed in a liquid can be found in the literature. O'Neill and coworker theoretically investigated the rotational and translational frictions for the special geometry of a bare spherical particle half- immersed in water (contact angle $\alpha_{\mathrm{eq}}=90^{\circ}$ ). ${ }^{18}$ Here the rotational friction coefficient $f_{r, \perp}$ describes a rotation of an angle $\beta$ (see Fig. 3) about an axis perpendicular to the interface normal as sketched in Fig. 4.

A non-dimensional sliding friction coefficient $B=R / b$, where $b$ is the slip length, has been introduced by O'Neill and coworker to evaluate the rotational friction. ${ }^{18}$ The case $B=\infty$ corresponds to the no-slip condition, whereas $B=0$ corresponds to the perfect slip condition. The rotational friction coefficient is: ${ }^{18}$

$f_{r, \perp}=k_{\perp}^{r} \eta R^{3}$,

where $\eta$ is the liquid viscosity. The expression of the friction factor $k_{\perp}^{r}$ is rather complex. It changes from zero in the perfect slip condition to infinity in the no-slip condition. The following empirical formula describes the asymptotic behavior for $B>1$ : 
Note that in the bulk the rotational friction is $8 \pi \eta \mathrm{R}^{3}$. A typical value of the slip length $b=1 \mathrm{~nm}(0.1$ $\mathrm{nm})^{41,42}$ for a colloidal particle of $R=1 \mu \mathrm{m}$ yields $B=1000$ (10000) and $k_{\perp}^{r}=33.6$ (43.9), which is $34 \%$ (75\%) higher than the bulk value. Hence the rotation of a bare colloid at the interface will be slowed down with a rotational diffusion time $\tau_{r, \perp}=f_{r, \perp} / k T=8.4 \mathrm{~s}(11.0 \mathrm{~s})$ significantly higher (but of the same order of magnitude) than the rotational diffusion time in the bulk water $\tau_{r, W}=6.3 \mathrm{~s}$.

For Janus colloids the scenario becomes more complicate because the free energy of the system changes significantly with the orientation (see Fig. 4). The rotational diffusion occuring in a harmonic potential can be described by an Arrhenius behavior:

$D_{r, \perp}=\frac{k T}{k_{\perp}^{r} \eta R^{3}} \exp \left(-\left|\Delta E_{\beta}\right| / k T\right)$,

where $\Delta E_{\beta}$ is the activation energy for the change of orientation. Note that if only thermal agitation $k T$ drives the rotation, the Janus colloid can theoretically rotate only in the region where the free energy keeps constant $\left(\alpha_{e q}<90^{\circ}-\beta<180^{\circ}-\alpha_{e q}\right)$ being the activation energy very high for typical values considered in Fig. 4.

Pinning of the contact line has also a strong impact on the dynamics of Janus colloids at the air-water interfaces. If strong defects are present, the contact line pinning leads to an additional friction and severe slowing down of the rotational diffusion. In fact, if the driving energy of rotation is only the thermal agitation $(k T)$, one expects a suppression of rotational motion if the defect energy $\gg k T$.

When the defect energy (i.e. the total energy dissipated by a single defect around a hysteresis cycle ${ }^{43}$ ) is comparable to $k T$, a slowing down of the rotational diffusion is also expected as it was observed experimentally for colloidal particle adsorbing or translating at the water interface and explained in terms of thermal hopping of the contact line. ${ }^{44,45}$

Janus colloid may rotate in the region of minimum free energy orientation but the contact line is expected to be bounded to the Janus boundary. It could even be completely stuck to the boundary since topological and chemical defects are expected to accumulate there. Hence, orientation $\beta=90^{\circ}-\alpha_{e q}$ or $-\left(\alpha_{e q}+90^{\circ}\right)$ may represent the most probable configurations, since the free energy is at the minimum and the contact line would be pinned, preventing any further rotation. 


\section{EXPERIMENTAL METHODS}

\subsection{PARTICLE FABRICATION}

Pt-SiO ${ }_{2}$ Janus colloids were fabricated following the procedure by Love et al. ${ }^{46}$ Silica beads (purchased from Microparticles $\mathrm{GmbH}$, radius $R=1.06 \pm 0.03 \mu \mathrm{m}$, zeta potential $=-13 \mathrm{mV}$ ) were cleaned by centrifugation/dilution cycles using Millipore water. This deionized water was produced by a Millipore Milli-Q filtration system with a resistivity of $18 \mathrm{M} \Omega \cdot \mathrm{cm}$.

First, a monolayer of silica beads was prepared on a silicon wafer (diameter $10 \mathrm{~cm}$ ) by drop-casting: drops of particle suspension at a concentration of $0.1 \mathrm{mg} / \mathrm{mL}$ were regularly deposited with a syringe onto a silicon wafer to reach an average coverage of about $10 \%$. Observation of the prepared sample shows that the particles form clusters of 4 to 30 particles rather than being homogeneously dispersed (as shown in Fig. 6A). This is possibly due to capillary force during the evaporation of residual water.

Using plasma bombarded metal sputtering (ALCATEL SCM 400 system), first $10 \mathrm{~nm}$ of titanium and then $20 \mathrm{~nm}$ platinum were deposited onto the silica beads monolayer. By 30 minutes' sonication, $\mathrm{Pt}$ coated silica beads were freed into Millipore water. These $\mathrm{Pt}_{-} \mathrm{SiO}_{2} \mathrm{Janus}$ colloids were then cleaned and collected by centrifugation/dilution cycles using Millipore water.

Scanning electronic microscopy (SEM, FEI Quanta 200F) was used to observe the as-prepared Janus colloids. The sample was prepared by making a drop of Janus colloid suspension onto silica wafer and dried. As shown in Fig. 6B, the SEM images show that the Pt-coated silica particle has two distinct faces. The white faces of the particles correspond to Pt-coated surfaces. The Janus boundary where the Pt-coated face and the bared silica face meet can be "linear" or "wavy". The wavy Janus boundary result probably from the shadowing effect during the metal deposition procedure. ${ }^{47}$ When silica beads are closely packed, as sketched in Fig. 6A, the nearby beads act as shields preventing the deposition of metal atoms.

Looking into the finer details of the Pt-coated face, as shown in Fig. 6C, the Pt layer presents some roughness at a characteristic length scale of about $10 \mathrm{~nm}$. When metal atoms are deposited onto a substrate which is rugged, the atoms do not arrive at the same time uniformly at the surface. This random fluctuation, which is inherent in the process, may create the surface roughness. 

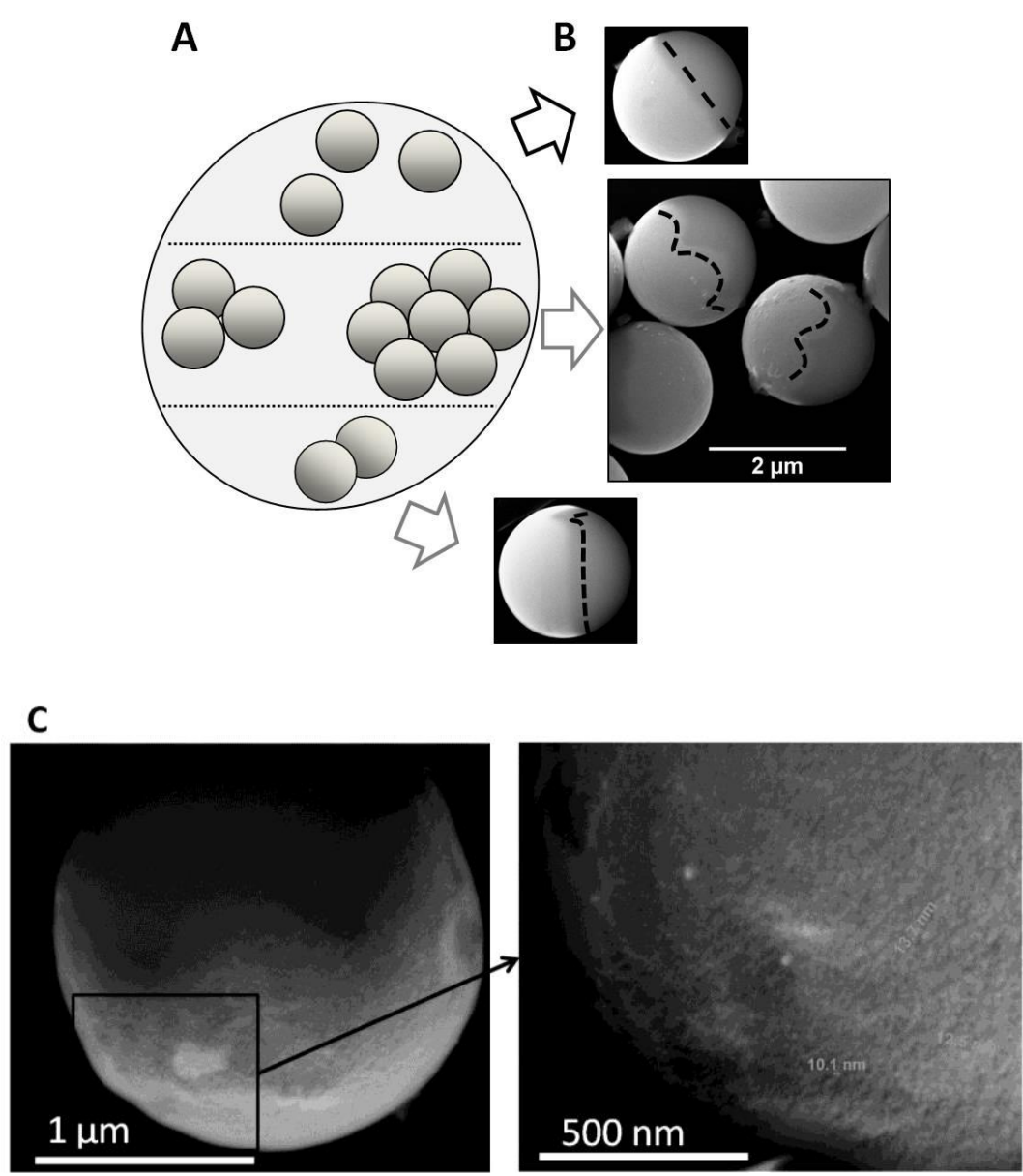

Fig. 6 (A) Sketch of silica beads monolayer on a silica wafer. (B) SEM images of $\mathrm{Pt}_{-\mathrm{SiO}}$ colloids. (C) SEM images of the Pt-coated surface.

\subsection{CONTACT ANGLE OF SINGLE JANUS COLLOIDS}

The contact angle of single colloid at the water surface was measured by an optical microscopy technique and a gel trapping method.

The immersion of colloids in water was measured by an in situ method as described by Hórvölgyi et $a l . .^{48}$ Janus colloids suspension in water was placed in between two parallel optical microscopic slides. The space between the two slides was $\sim 4 \mu \mathrm{m}$. Some Janus particles attach onto the air-water interface. For observation, sample cell was laid down under an inverted microscope equipped with an oil-phase objective (magnification $\times 100$ ).

Due to the fact that the water layer between the two slides is rather thin, evaporation results in contact line motion of the water suspension on the glass slides. Concomitant motion of the particle demonstrated that particles were trapped at the air-water interface and not stuck onto the glass wall.

Another indirect measurement of the particle-water contact angle is to use a gel trapping method. ${ }^{49} \mathrm{~A}$ $2 \mathrm{wt} \%$ Gellen (Phytagel, Sigma-Aldrich) solution in Millipore water was prepared by heating at $90^{\circ} \mathrm{C}$ under magnetic stirring. After cooling down to room temperature the gel was formed (gel point 27$32^{\circ} \mathrm{C}$ ). Beads suspension in water was spread at the gel surface and again heated at $90^{\circ} \mathrm{C}$ to let particles be trapped at the interface. Immersion depth of beads at gelled water surface is expected to be similar to the one at liquid water surface since the two interfaces have the same surface tension. 
To get a replica of the gel interface with the beads, Norland Optical Adhesive 81 (NOA81) was poured over the gelled water surface and then photopolymerized by ultraviolet light for 2 minutes. The solidified NOA81 layer was peeled off taking particles at complementary positions with respect to that at air-gelled water interface. These samples were finally observed by both optical microscopy and Scanning Electron Microscopy.

\subsection{SESSILE DROP CONTACT ANGLE ON PLANAR SURFACES}

We measured contact angles of macroscopic water sessile drops with planar silica and Pt-coated surfaces. The planar silica surface is the one of a silicon wafer and the platinum coating was made on the same silicon wafer surface. Silicon wafers were first cleaned with ethanol and acetone, and finally with Millipore water. The Pt-coated planar surface was prepared the same way as that for Janus colloids by using metal deposition method. ${ }^{15}$ First $10 \mathrm{~nm}$ of titanium was deposited onto the wafer and then $20 \mathrm{~nm}$ platinum was deposited. The droplet profile was measured using a profile analyzer tensiometer (PAT, Sinterface, Germany).

\subsection{PARTICLE TRACKING AND IMAGE ANALYSIS}

Tracking of isolated particles was achieved by using a Basler Scout CCD camera equipped Leica inverted microscope mounted on a Melles Griot optical table and a Leica objective of magnification $\times 32$. The video with a field of view $125 \mu \mathrm{m} \times 94 \mu \mathrm{m}$ was recorded at a rate of 30 frames per second. The tracking was performed under Labview (National Instruments) using an image correlation-based approach ("Stat Tracker St. Andrews") to obtain the particle position over time [time t (s), x $(\mu \mathrm{m}), \mathrm{y}$ $(\mu \mathrm{m})]$. Using IDL software, we treated raw image sequences and by inputting a threshold on the grayscale level we detected the Pt-cap. Counting the number of elements in pixel, the area of Pt-cap detected could be evaluated.

\section{EXPERIMENTAL RESULTS AND DISCUSSION}

\subsection{JANUS COLLOID CONTACT ANGLE AND ORIENTATION}

Representative optical microscopy images displaying the immersion depth and orientation of Janus colloid at the air-water interface are shown in Fig. 7. The black region of the Janus colloid is the platinum face.

We evaluated the contact angle $\alpha$ of the particle from the image by using the software ImageJ (and the "Contact Angle" plug-in) as shown in Fig. 7. The accuracy of the method is about $10^{\circ}$ in the range of contact angle considered here. 


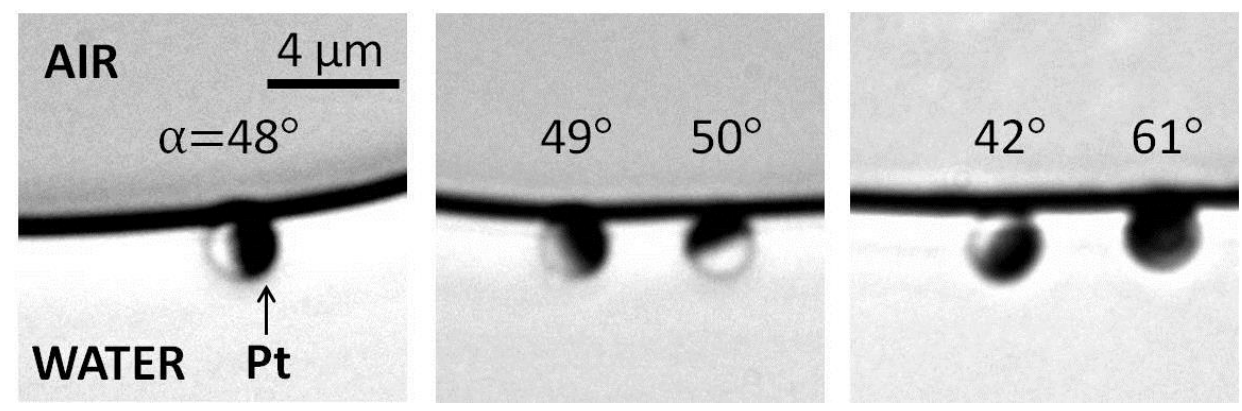

Fig. 7 Microscopic images of Janus colloids at the air-water interface. The black surfaces of colloids are coated with Pt. Contact angles $(\alpha)$ measured by image analysis are shown.

Since the Janus colloid attached onto the interface from water, where they were originally dispersed, the contact angle measured in Fig. 7 can be regarded as the receding contact angle of the Janus colloids. The average contact angle measured for different Janus colloids is $50^{\circ}$ with a standard deviation of $6^{\circ}$.

We observed different possible orientations of the Janus colloids. This result is somewhat surprising if considering that literature equilibrium contact angle of platinum is lower than the one of silica. ${ }^{24,26,25}$ As pointed out in section 2.1 and 2.2 for literature values of the interfacial intensions of silica and platinum one expects the platinum face to be all wetted by water.

In Fig. 8 SEM images of Janus colloid at the interface of solidified NOA81 layer are also shown. Note that the visible part of colloid is the one previously immersed in gelled water. Since Janus colloids were deposited on top of the gelled water and hence attached onto the interface from air, the contact angle measured in Fig. 8 can be considered as the advancing contact angle of the Janus colloids, $\alpha_{\mathrm{JANUS}, \mathrm{A}}=64 \pm 2{ }^{\circ} \cdot{ }^{15}$ Experimental contact angles $\alpha_{\mathrm{JANUS}, \mathrm{R}}=50^{\circ} \pm 6^{\circ}$ and $\alpha_{\mathrm{JANUS}, \mathrm{A}}=64 \pm 2$ can be compared to the theoretical equilibrium contact angles of silica, $\alpha_{S, e q}=61^{\circ}$, and platinum, $\alpha_{P, e q}=52^{\circ}$, shown in Figure 2 (B).

The orientation of Janus colloids also shows a variety of ranges. Some Janus colloids were straddling the water surface with the air-water interfacial plane across their Janus boundaries. This can be seen clearly from the SEM image shown in Fig. 8. Elemental analysis was used to qualitatively assess that the region of high Pt \% correspond to the brighter face of the Janus colloid. For some other Janus colloids, the interfacial plane can even cross only the Pt-coated surface (see the right SEM image in Fig. 8). Note that we did not observe any significant pinning of the contact line on the Janus boundary, see Fig. 7 and 8. Hence the "wavy" form of the Janus boundary cannot be invoked to explain the orientation of the Janus colloid at the surface. 


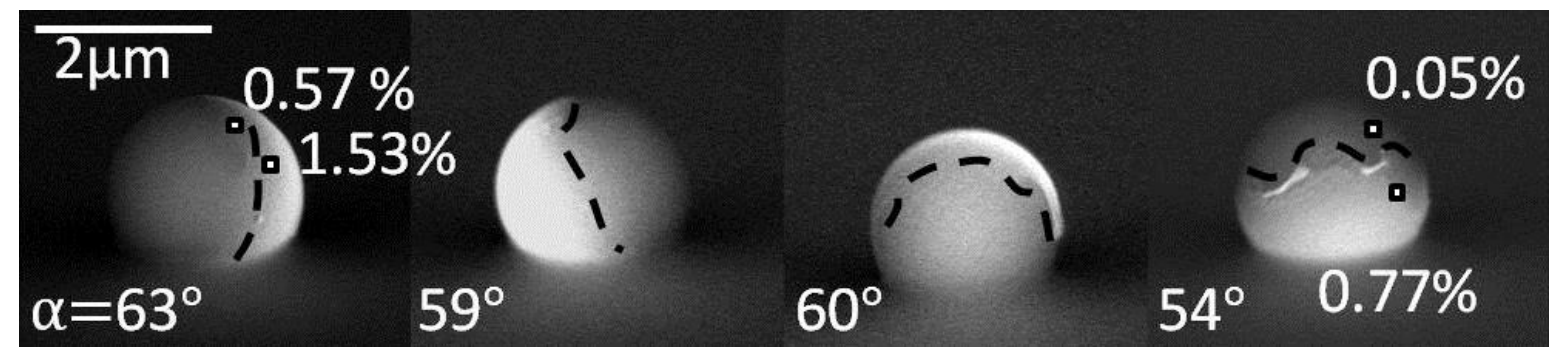

Fig. 8 SEM images of Pt-SiO 2 beads trapped in the NOA81 layer. Pt atomic percentages are shown for two particles with the white parts corresponding to Pt-coated surfaces.

Random orientation was observed for other Janus systems such as gold-polystyrene particles at the oilwater interface ${ }^{50}$ or gold-silica particles at the air-water and oil-water interfaces. ${ }^{51}$ It was believed that difference in equilibrium contact angles could reveal the difference of free energy for different orientations ${ }^{50}$ and the effect of pinning and surface roughness was qualitatively discussed.

Here we measure in Figure 7 and 8 an hysteresis of Janus colloid contact angle:

$\Delta \cos \alpha_{J A N U S}=\cos \alpha_{J A N U S, R}-\cos \alpha_{J A N U S, A}=0.2$, which points to a significant pinning of the contact line on surface defects, see section 2.3. These defects are clearly observed on the platinum coating as surface nanoroughness and on the Janus boundary. Note also that we did not find any significant change of the contact angle with the orientation of the Janus colloid. Thus, contact line pinning affects strongly the interfacial behavior of Janus colloids and the energy profiles based on ideal surfaces with single equilibrium contact angles cannot fully describe our results. In this sense, future theoretical investigations should take into account the influence of the contact angle hysteresis in the free energy.

\subsection{EFFECT OF PLATINUM COATING ON WETTING}

In order to further check the effect of contact line pinning we measured the liquid drop contact angles on solid planar surfaces. We aimed at investigating the effect of the coating layer on a silica substrate.

Results are listed in Table 1. It shows that the advancing angle of water with the platinum coated surface is slightly higher than with silica surface, while receding contact angles show the opposite trend.

Table 1 Contact angles of the liquid drop with silica surface and Pt-coated surface.

\begin{tabular}{|l|c|c|}
\hline Contact angle & $\begin{array}{c}\text { Advancing } \\
\left(\boldsymbol{\alpha}_{A}\right)\end{array}$ & $\begin{array}{c}\text { Receding } \\
\left(\boldsymbol{\alpha}_{\boldsymbol{R}}\right)\end{array}$ \\
\hline Water-Silica surface & $56.9 \pm 1.0^{\circ}$ & $44.3 \pm 0.2^{\circ}$ \\
\hline $\begin{array}{l}\text { Water-Pt-coated } \\
\text { surface }\end{array}$ & $59.1 \pm 1.3^{\circ}$ & $40.1 \pm 0.4^{\circ}$ \\
\hline $\begin{array}{l}\text { Heptane-Silica } \\
\text { surface / Pt-coated } \\
\text { surface }\end{array}$ & 0 & 0 \\
\hline
\end{tabular}

For other high surface tension liquids like glycerol we observed the same increase of contact angle hysteresis $\Delta \cos \alpha$ for the coating layer. Instead low surface tension liquids like heptane show complete 
wetting. Note also that the absolute values of the advancing and receding contact angles are not very different between the bare silica and the platinum coated surface. Hence the energy of the two surfaces are somewhat similar. However as we pointed out before, hysteresis of the contact angle $\Delta \cos \alpha_{\text {SILICA }}=0.16$ and $\Delta \cos \alpha_{\text {PT-COAT }}=0.25$ reveals the presence of contact line pinning on strong defects. Using equation 9 , and assuming the geometrical factor $a=1$ and $\Delta \cos \alpha=0.2$, a defect density $\phi=0.4$ is calculated. Note that for this defect density (40\%) the energy profile may change dramatically, see Figure 5.

\subsection{ROTATIONAL FRICTION OF BROWNIAN JANUS COLLOIDS AT THE AIR-WATER INTERFACE}

The presence of surface defects as discussed in section 2.4 may also significantly slows down the rotational diffusion of the Janus colloid. Therefore, the orientation of a Janus colloid once attached onto the interface may not change significantly with time if the contact line is strongly pinned.

By analyzing the images taken during the tracking of the Brownian motion of Janus colloids at a planar air-water interface, we were able only in few cases to evaluate the area of the platinum face observed by optical microscopy from the top. Hence, we calculate the orientation angle $\beta$ from the area of the platinum face obtained by image analysis, and plot the mean square angular displacement MSAD in Fig. 9 for one specific dataset for which $40^{\circ}<\beta<90^{\circ}$.

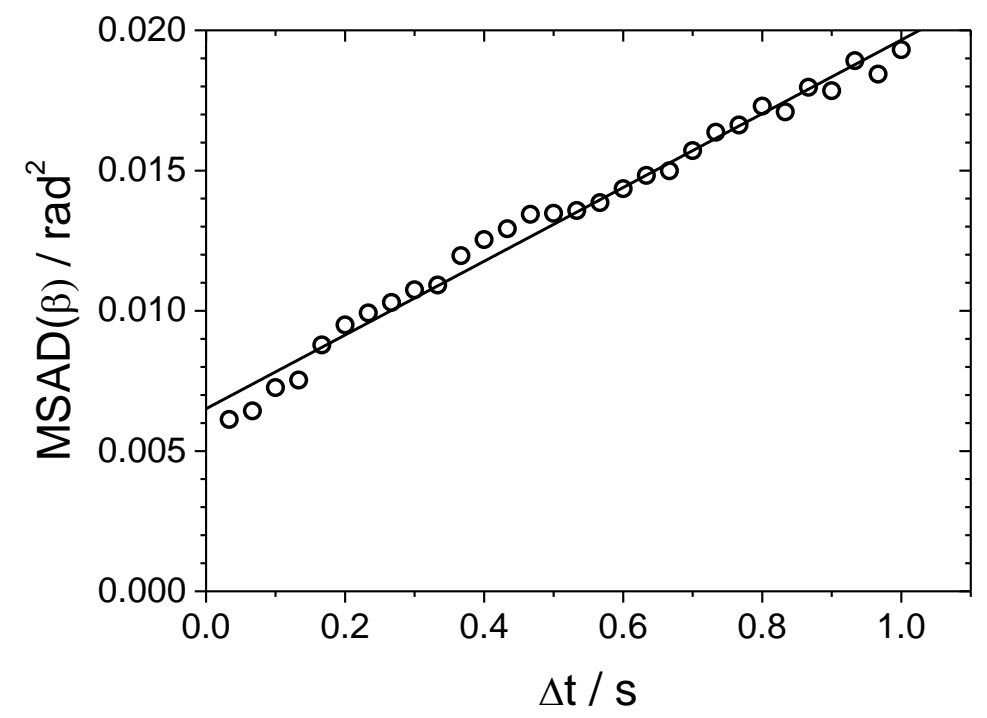

Figure 9. Mean squared angular displacement as a function of the lag time for a Brownian Janus colloid diffusing at the air-water interface.

We fitted the MSAD results by $\operatorname{MSAD}(\beta)=\operatorname{MSAD}_{0}+2 \mathrm{D}_{\mathrm{r}, \perp} \Delta \mathrm{t}$, where the $\mathrm{MSAD}_{0}$ accounts for the error introduced by the image treatment. Rotational diffusion time $\tau_{\mathrm{r}, \perp}=1 / \mathrm{D}_{\mathrm{r}, \perp}=152 \mathrm{~s}$ measured in Fig. 9 is much larger than the time predicted accounting only for hydrodynamic frictions, $\tau_{\mathrm{r}, \perp}=8.4 \ldots 11.0 \mathrm{~s}$, see section 2.4. Note also that an energy $\Delta E_{\beta}$ of only few $k T$ in eq. 8 could explain the experimental rotational diffusion time $\tau_{\mathrm{r}, \perp}=152 \mathrm{~s}$. However, for the angular displacement shown in Fig. 9, one expects $\Delta E_{\beta} \approx 10^{5} k T$ in the ideal case shown in Fig.4. 
As discussed in section 2.3, a slowing down of the rotational diffusion can be attributed to the dynamics of pinning-depinning of the contact line in presence of topographic defects as the platinum face shows roughness in the nanometric scale (see Fig. 6). ${ }^{34,44}$

\section{SELF-PROPELLED JANUS COLLOIDS AT THE SURFACE OF WATER}

Catalytic Janus colloids attached onto at the water surface may show autonomous motion if a reactant is present in the aqueous phase as fuel. For platinum coated Janus colloids, the fuel is hydrogen peroxide and concentrations of few percent are enough to show directional trajectories and speed of 10 $\mu \mathrm{m} / \mathrm{s} .{ }^{15}$ To realize self-propulsion parallel to the interface plane several requirements have to be fulfilled. In this section, theoretical and experimental results shown before will be used to discuss the ability of Janus colloids to perform self-propulsion at the surface of water.

First, the largest contact area between the catalytic surface and the liquid containing the fuel is seeked. Furthermore the Janus boundary should not remain parallel to the interface, since no propulsive force can then be generated in a direction parallel to the interfacial plane. The latter requirement excludes amphiphilic Janus colloids to perform self-propulsion parallel to the interface. In fact, as discussed in section 2.1, the Janus boundary of amphiphilic Janus colloids remains parallel to the interface and the equilibrium contact angle is $90^{\circ}$, see Fig. 1. The double hydrophilic Janus colloid instead is able change its orientation between the limiting cases where the Janus boundary touches the air-water interface without increasing its free energy. In fact, all these configurations result in the same free energy of the system, see Fig. 4. Hence if the colloids' surfaces are defect free, double hydrophilic colloids with low equilibrium contact angles are expected to show a better ability to self-propel at high speed parallel to the interface with respect to double hydrophilic colloids with high equilibrium contact angles. In this regard, a Janus micrometric colloid with the catalytic $P$ face possessing $\alpha_{P, e q}=0$ and $\alpha_{S, e q}$ of few degrees shows the optimal conditions for the realization of self-propulsion at the surface of water. Note that when both faces of the Janus colloid possess the same equilibrium contact angle $\alpha_{P, e q}=\alpha_{S, e q}$ the colloid is equivalent to a bare colloid and all orientation correspond to the same energy. In the latter system for relatively high contact angles, one expects an active motion interrupted by a thermal Brownian motion corresponding to the orientations for which the catalytic face is pulled out from the aqueous phase. Finally, the presence of the fuel may affect the interfacial tensions and the free energy landscape as well as the overall dynamics may depend non trivially on the fuel concentration. ${ }^{52}$ Note that for hydrogen peroxide solutions the surface tension change is very small. ${ }^{53}$

Experimental observations for silica-platinum Janus colloids presented in section 4 show the importance of surface roughness, which leads to contact line pinning resulting in contact angle hysteresis and random orientations observed at the interface. Pinning of the contact line plays also a crucial role for the slowing down of the rotational diffusion, see section 2.4 and 4.3. Contact line pinning over defects present on the Janus colloid surface may be invoked to explain the slowing down of the rotational diffusion observed in the absence of catalytic fuel. This result agrees also with the observation that in presence of hydrogen peroxide, the propulsion speed keeps constant with time, which corresponds to a small variation of the orientation angle $\beta .^{15}$

\section{CONCLUSION}

We have shown that the behavior of a Janus particle trapped at the air-water interface is controlled by the wettability of its two faces. At equilibrium, stable configurations can be determined by minimizing 
the free energy of the system upon variation of the relative orientation of the Janus boundary with respect to the interface normal. By assuming a flat interface, we have been able to derive an analytical expression for the dependence of the free energy as a function of the orientation. It is worth noting that our model provides the exact free energy difference between states in which both halves of the particle are wetted by a single fluid phase, hence providing bounding values for the free energy once deformation of the interface is taken into account.

Comparing our analytical results to our experiments we realized that contact line pinning play crucial roles for the description of the interfacial behavior of Janus colloids. In particular, contact line pinning due to surface roughness or defects probably prevents the predicted equilibrium position and orientation at the air-water interface to be reached. Surface roughness and defects, which are more dense at the Janus boundary and on the catalytic face, cause random orientation of Janus colloids at the interface. Surface defects also slow down significantly the rotational diffusion of the Janus colloid.

Our results shed light on the behavior of Janus colloids self-propelling at the air-water interface. Janus colloids at the fluid interface may behave as kinetically driven system, where the contact line motion over defects decorating the Janus faces rules the contact angle hysteresis, orientation and rotational diffusion of the particle. Such a feature is crucial not only for the dynamics of a single particle, but also for their collective behavior and therefore for the development of self-assembly at fluid-fluid interfaces.

\section{ACKNOLDEGEMENTS}

We thank Michel Gross for discussions and helping us with some calculations. Financial supports from Labex Chemisyst and Labex Numev are also acknowledged.

\section{REFERENCES}

1. A. Walther and A. H. E. Müller, Soft Matter, 2008, 4, 663.

2. S. Jiang, Q. Chen, M. Tripathy, E. Luijten, K. S. Schweizer, and S. Granick, Adv. Mater., 2010, 22, 1060-1071.

3. A. Perro, S. Reculusa, S. Ravaine, E. Bourgeat-Lami, and E. Duguet, J. Mater. Chem., 2005, 15,3745 .

4. Q. Chen, J. K. Whitmer, S. Jiang, S. C. Bae, E. Luijten, and S. Granick, Science, 2011, 331, 199-202.

5. T. Ondarçuhu, P. Fabre, E. Raphaël, and M. Veyssié, J. Phys., 1990, 51, 1527-1536.

6. C. Casagrande, P. Fabre, E. Raphaël, and M. Veyssié, Europhys. Lett., 2007, 9, 251-255.

7. S. Fujii, M. Kappl, H.-J. Butt, T. Sugimoto, and Y. Nakamura, Angew. Chem. Int. Ed. Engl., 2012, 51, 9809-13. 
8. R. Aveyard, Soft Matter, 2012, 8, 5233.

9. A. Kumar, B. J. Park, F. Tu, and D. Lee, Soft Matter, 2013, 9, 6604-7202.

10. R. Golestanian, T. B. Liverpool, and A. Ajdari, New J. Phys., 2007, 9, 126.

11. W. Wang, W. Duan, S. Ahmed, T. E. Mallouk, and A. Sen, Nano Today, 2013, 8, 531-534.

12. J. Howse, R. Jones, A. Ryan, T. Gough, R. Vafabakhsh, and R. Golestanian, Phys. Rev. Lett., 2007, 99, 048102.

13. B. Jurado-Sánchez, S. Sattayasamitsathit, W. Gao, L. Santos, Y. Fedorak, V. V Singh, J. Orozco, M. Galarnyk, and J. Wang, Small, 2014, 11, 499-506.

14. L. Soler, V. Magdanz, V. M. Fomin, S. Sanchez, and O. G. Schmidt, ACS Nano, 2013, 7, 9611-20.

15. X. Wang, M. In, C. Blanc, M. Nobili, and A. Stocco, Soft Matter, 2015, 11, 7376-7384.

16. P. Malgaretti, M. N. Popescu, and S. Dietrich, Soft Matter, 2016, DOI: 10.1039/c6sm00367b.

17. R. Golestanian, T. Liverpool, and A. Ajdari, Phys. Rev. Lett., 2005, 94, 1-4.

18. M. E. O’Neill, K. B. Ranger, and H. Brenner, Phys. Fluids, 1986, 29, 913.

19. H. Rezvantalab, G. Drazer, and S. Shojaei-Zadeh, J. Chem. Phys., 2015, 142

20. J. Drelich, Colloids Surfaces A Physicochem. Eng. Asp., 1996, 116, 43-54.

21. L. Schimmele, M. Naplórkowski, and S. Dietrich, J. Chem. Phys., 2007, 127, 164715.

22. T. Getta and S. Dietrich, Phys. Rev. E, 1998, 57, 655-671.

23. J. G. Gibbs and Y.-P. P. Zhao, Appl. Phys. Lett., 2009, 94, 163104.

24. B. P. Binks and J. H. Clint, Langmuir, 2002, 18, 1270-1273.

25. V. Leo, A. Tusa, Y. C. Araujo, and V. Leo, 1999, 155, 131-136.

26. J. R. Gardner and R. Woods, J. Electroanal. Chem. Interfacial Electrochem., 1977, 81, 285290.

27. J. Faraudo and F. Bresme, J. Chem. Phys., 2003, 118, 6518-6528.

28. H. Lehle, E. Noruzifar, and M. Oettel, Eur. Phys. J. E, 2008, 26, 151-160.

29. H. Rezvantalab and S. Shojaei-Zadeh, Soft Matter, 2013, 9, 3640.

30. D. L. Cheung and S. a. F. Bon, Soft Matter, 2009, 5, 3969.

31. B. J. Park and D. Lee, ACS Nano, 2012, 6, 782-90. 
32. J. F. Joanny and P. G. de Gennes, J. Chem. Phys., 1984, 81, 552.

33. V. Hisler, L. Vonna, V. Le Houerou, S. Knopf, C. Gauthier, M. Nardin, and H. Haidara, Langmuir, 2014, 30, 9378-83.

34. S. Ramos and a Tanguy, Eur. Phys. J. E. Soft Matter, 2006, 19, 433-40.

35. D. Bonn and G. Wegdam, J. Phys., 1992, 2, 1755.

36. T. D. Blake, J. Colloid Interface Sci., 2006, 299, 1-13.

37. M. Reyssat and D. Quéré, J. Phys. Chem. B, 2009, 113, 3906-3909.

38. R. N. Wenzel, J. Ind. Eng. Chem. (Washington, D. C.), 1936, 28, 988-994.

39. D. Bonn, J. Eggers, J. Indekeu, J. Meunier, and E. Rolley, Rev. Mod. Phys., 2009, 81, 739-805.

40. D. L. Cheung, Chem. Phys. Lett., 2010, 495, 55-59.

41. P. G. de Gennes, Langmuir, 2002, 18, 3413-3414.

42. C. Sendner, D. Horinek, L. Bocquet, and R. R. Netz, Langmuir, 2009, 25, 10768-81.

43. J.-M. Di Meglio and D. Quéré, Europhys. Lett., 2007, 11, 163-168.

44. G. Boniello, C. Blanc, D. Fedorenko, M. Medfai, N. Ben Mbarek, M. In, M. Gross, A. Stocco, and M. Nobili, Nat. Mater., 2015, 14, 908-11.

45. D. M. Kaz, R. Mcgorty, M. Mani, M. P. Brenner, and V. N. Manoharan, Nat. Mater., 2012, 11, $138-42$.

46. J. C. Love, B. D. Gates, D. B. Wolfe, K. E. Paul, and G. M. Whitesides, Nano Lett., 2002, 2, 891-894

47. T. Karabacak, J. Nanophotonics, 2011, 5, 052501.

48. S. N. Z. Hórvölgyi J. H. Fendler, Colloids Surf. A Physicochem. Eng. Asp., 1993, 71, 327.

49. V. N. Paunov, Langmuir, 2003, 19, 7970-7976.

50. B. J. Park, T. Brugarolas, and D. Lee, Soft Matter, 2011, 7, 6413.

51. D. J. Adams, S. Adams, J. Melrose, and A. C. Weaver, Colloids Surfaces A Physicochem. Eng. Asp., 2008, 317, 360-365.

52. A. Domínguez, P. Malgaretti, M. N. Popescu, and S. Dietrich, Phys. Rev. Lett., 2016, 116, 078301.

53. M. Phibbs and P. Giguere, Cana. J. Chem, 1951, 29, 173. 